\title{
APLICAÇÃO DO TRIÂNGULO DE PASCAL
}

\author{
ALEAE INTERRUPTAE: A CURIOUS APLICATION OF PASCAL'S \\ TRIANGLE
}

\author{
John A. Fossa ${ }^{1}$ \\ Dept ${ }^{\circ}$. de Matemática, PPGED, \\ PPGECEM da UFRN (aposentado)
}

\section{Resumo}

O presente artigo objetiva, através de uma análise linguística e matemática, fazer uma breve explanação de certos aspetos do Triângulo Aritmético (Triângulo de Pascal), conforme a abordagem de Blaise Pascal (1623-1662) no seu Tratado sobre o Triângulo Aritmético. Especificamente, investiga-se uma das várias aplicações do referido Triângulo, a saber, como dividir a aposta de um jogo de soma zero, caso for necessário interromper o mesmo antes de chegar à sua conclusão. Apresenta-se um método para fazer a divisão sem usar o Triângulo (como apresentado por Pascal) e, então, o método de Pascal por fazer a mencionada divisão usando o Triângulo, pois a demonstração deste método depende daquele. Finalmente, analisa-se a demonstração dada por Pascal de que o seu método usando o Triângulo é válido, pois a referida demonstração é uma das primeiras na história em que a Indução Matemática foi formulada corretamente. Concluímos que a formulação do argumento por Indução Matemática é, de fato, correta e que a base da indução é bem-feita. O passo da indução, ou seja, a passagem do $n$ ésimo caso para o $n+1$-ésimo caso, porém, não é válido pelos padrões lógicos modernos porque não é feito com a generalidade necessária. Também concluímos, porém, que Pascal certamente considerava sua demonstração válida, pois considerava o caso abordado como um template que é aplicável a todos os casos da demonstração.

Palavras-chave: História e Filosofia da Matemática; Educação Matemática; Triângulo de Pascal; Demonstração Matemática.

\section{Abstract}

The present article proposes to make, by a linguistic and mathematical analysis, a brief explanation of certain aspects of the Arithmetic Triangle (Pascal's Triangle), as it was set out by Blaise Pascal (1623-1662) in his Treatise on the Arithmetic Triangle. Specifically, one of the various applications of the aforementioned Triangle, that of how to divide a wager in a zero sum game, when it is necessary to interrupt the game before reaching its conclusion, is analyzed. Following Pascal, a method for making the division without using the Triangle is presented first and then Pascal's method of making the division using the Triangle is set out, since the demonstration of the latter depends on that of the former. Finally, the demonstration of the validity of Pascal's method of

\footnotetext{
${ }^{1}$ Endereço eletrônico: jfossa@oi.com.br.
} 
making the division is analyzed, given that this demonstration is one of the first in history in which Mathematical Induction is formulated correctly. We conclude that the formulation of the argument by Mathematical Induction is indeed well formulated and that the base of the induction is correct. The induction step, that is, the passage from the $n$th case to the $n+1^{\text {st }}$ case, however, is not valid according to modern standards of logic, because it is not done with the necessary level of generalization. We also conclude, however, that Pascal certainly considered his argument valid because he considered the case given by him to be a template that could be applied to all the cases in the Triangle.

Keywords: History and Philosophy of Mathematics; Mathematics Education; Pascal's Triangle; Mathematical Demonstration.

\section{Introdução}

O Triângulo de Pascal é um conhecido arranjo triangular de números, discutido por Blaise Pascal (1623-1662) no seu Tratado sobre o Triângulo Aritmético (Pascal, 2013). O Triângulo não se originou com Pascal, mas foi conhecido por vários matemáticos em várias culturas desde a antiguidade. Mesmo assim, Pascal não só desenvolveu o conceito do Triângulo independente dos seus predecessores, mas também deu, em Pascal (2013), uma descrição detalhada do mesmo, bem como um relato de várias das suas aplicações. No presente trabalho, abordaremos uma dessas aplicações, a saber, como fazer a divisão da aposta num jogo de azar, caso seja necessário interromper o jogo antes de chegar ao fim dele. Dedicaremos bastante atenção à demonstração dada por Pascal do procedimento por ele apresentado de fazer a referida divisão, pois é uma das primeiras vezas na história que uma demonstração por Indução Matemática é corretamente formulada.

\section{O Triângulo de Pascal}

O Triângulo é representado de formas diferentes por autores diferentes. A Figura 1 mostra o Triângulo como foi dado por Pascal. Hoje em dia o Triângulo é geralmente elaborado de forma girada, fazendo com que as diagonais da Figura 1 fiquem na horizontal. Isto é interessante no sentido de que a terminologia de Pascal para as diagonais ("bases") parece mais apropriada para o Triângulo girado. Observamos também que Pascal não usa a terminologia "Triângulo de Pascal", mas a expressão "Triângulo Aritmético". No restante do presente trabalho, seguiremos Pascal e utilizaremos a nomenclatura "Triângulo Aritmético". 
O Triângulo Aritmético é determinado por um valor inicial (o da primeira diagonal, o único que contém uma única célula) e uma regra de formação. $O$ valor inicial é geralmente dado como 1; embora Pascal contemple outros valores iniciais, só utiliza a unidade no seu Tratado. A regra de formação é a seguinte: o valor de uma nova célula é a soma do valor da célula que o procede na mesma linha ("fileira paralela" na terminologia de Pascal) e o valor da célula que o procede na mesma coluna ("fileira perpendicular" na terminologia de Pascal). Quando não há uma célula precedente na mesma linha, o valor da célula precedente da mesma coluna é repetido; semelhantemente para o caso em que não há uma célula precedente da mesma coluna.

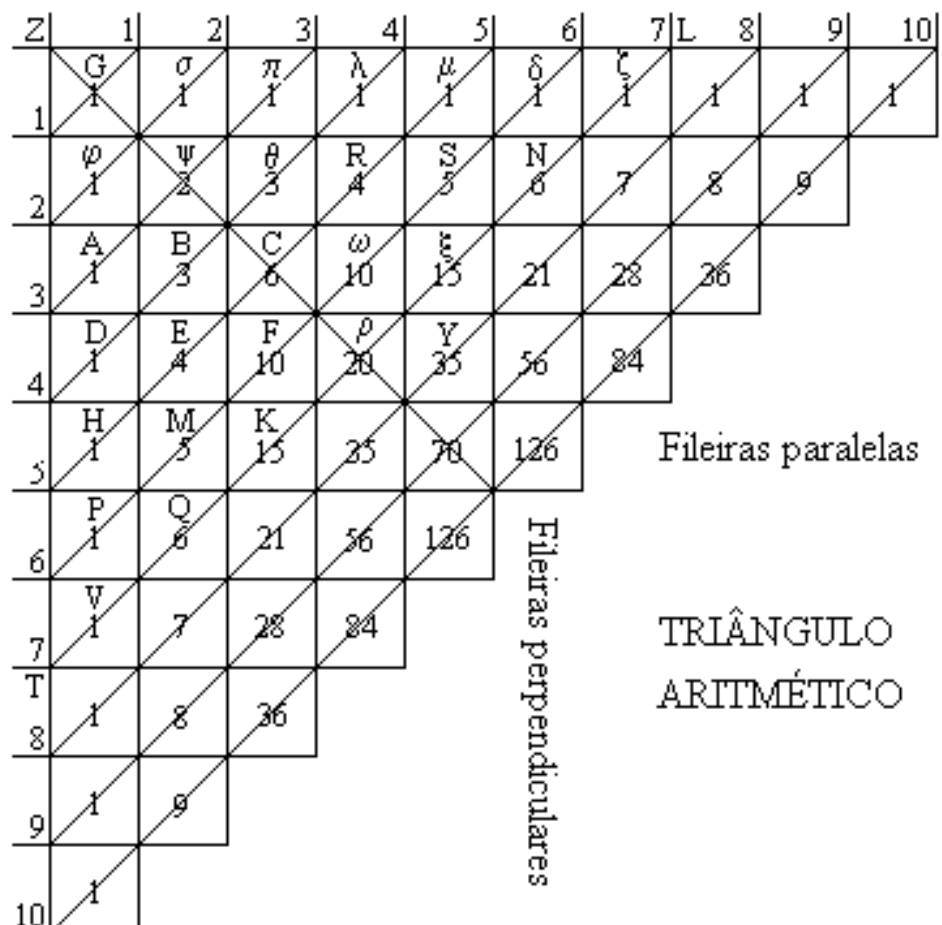

Figura 1. O Triângulo Aritmético.

Fonte: Pascal (2013, p. 54).

\section{O Jogo}

O tipo de jogo que Pascal contempla é um jogo entre dois jogadores, em que há várias partidas, sendo vitorioso aquele que ganhar um número estipulado, $n$, de partidas. Em princípio, cada partida deverá ter um, e apenas um, ganhador (zero sum game, “jogo de soma zero"), mas devido ao fato de que a natureza material das partidas não importa, podemos generalizar um pouco. Assim, por exemplo, podemos contemplar partidas 
John A. Fossa

Aplicação do triângulo de Pascal

empatadas, uma vez que os empates podem ser descartados, ou seja, podemos não os levar em conta na computação do número $n$. No que segue, entretanto, podemos ignorar essas complicações, pois a análise não será modificada.

Caso o jogo proceder normalmente até um dos jogadores alcançar as $n$ partidas ganhas, não terá problema. Mas, Pascal contempla a situação em que o jogo tiver de ser interrupto antes de alguém alcançar as $n$ partidas. Neste caso, pergunta-se: como a aposta deve ser dividida? Em resposta, mostra que a divisão depende, não do número de partidas ganhas por cada jogador, mas pelo número de partidas que falta a cada jogador para ganhar o jogo; ainda mostra que isto pode ser calculado usando o Triângulo Aritmético. Assim, formula o problema da seguinte maneira (Pascal, 2013, p. 90, itálico no original):

Seja proposto que há dois jogadores e a cada um falta um certo número de partidas para fechar o jogo. Ache, usando o Triângulo aritmético, as partes que devam ser feitas (se eles queiram separar sem jogar), levando em consideração as partidas que faltam para cada um.

Observamos que Pascal não pede resolver a questão da divisão da aposta em termos gerais, mas apenas como resolver o problema usando o Triângulo Aritmético. De fato, ele já havia resolvido o problema, nas páginas anteriores do seu tratado, sem usar o Triângulo. Seu plano agora é mostrar como o mesmo pode ser efetuado com o Triângulo Aritmético e demonstrar que o procedimento está de acordo com a resposta geral.

O procedimento é então descrito da seguinte maneira (Pascal, 2013, p. 90), onde "bases" refere-se às diagonais do Triângulo:

Seja tomada, no triângulo, a base em que há o mesmo número de células quanto partidas que faltam para os dois jogadores juntos. Em seguida, seja tomado, nessa base, o mesmo número de células contíguas, começando com a primeira, que faltam para o primeiro jogador e faça a soma dos seus números. Assim, a quantidade das outras células serão a mesma que a das partidas que faltam ao outro jogador. Toma-se também a soma dos seus números. Essas somas estão uma para a outra como os recíprocos das vantagens dos jogadores. Desta forma, se a quantia que está em jogo é igual à soma dos números de todas as células da base, pertencerá a cada um o que é contido na quantidade de células que é a mesma de partidas que faltam para o outro. E se houver outra quantia em jogo, suas partes serão em essa proporção. 
Para entender melhor o procedimento, vejamos um exemplo. Supõe-se que há dois jogadores, A e B, que precisam interromper um jogo de soma zero, em que cada um apostou 100 reais. Na hora da interrupção, A necessita de apenas duas partidas para ganhar o jogo, enquanto B precisa de quatro partidas. Visto que o número total de partidas necessárias são $2+4=6$, tomamos a sexta diagonal (que consiste de 6 células). Mas, segundo Pascal, o número de células necessárias de cada jogador está a recíproca da sua expetativa de ganhar o jogo. Assim, para A, somamos os valores nas primeiras quatro (o número de partidas necessitadas de B) células da sexta diagonal e, para B, somamos os valores nas duas (o número de partidas necessitadas de A) células restantes. ${ }^{2}$ Temos, desta forma, para $\mathrm{A}, 1+5+10+10=26 \mathrm{e}$, para $\mathrm{B}, 5+1=6$. Visto que a soma de todos os valores das seis células da referida diagonal é 32 , A receberá $\frac{26}{32}=\frac{13}{16}$ da aposta, enquanto B receberá $\frac{6}{32}=\frac{3}{16}$ da aposta. Mas, a aposta total foi de 200 reais. Assim, A receberá $\frac{13}{16} \times 200=162,50$ reais, enquanto $B$ receberá $\frac{3}{16} \times 200=37,50$ reais.

\section{Interlúdio}

Antes de proceder para a demonstração do procedimento de Pascal, precisamos compreender como ele calcula a referida divisão sem usar o Triângulo Aritmético. Para tanto, ele usa os seguintes três princípios:

1. Se a um jogador não faltar qualquer partida, enquanto ainda falta qualquer número de partidas para o outro, o primeiro ganhará a aposta inteira;

2. Se aos dois jogadores faltarem o mesmo número de partidas, cada um ganhará metade da aposta;

3. Se os dois jogadores A e B estiveram em condições tais que A receberá $\frac{x}{z}$ da aposta, caso ganhe a próxima partida, e receberá $\frac{y}{z}$ da aposta caso perca a próxima partida, então, ao interromper o jogo (sem fazer a próxima partida), A ganhará $\frac{x+y}{2 z}$ da aposta.

\footnotetext{
${ }^{2}$ Visto que as diagonais são simétricas, podemos começar com a sexta célula da primeira coluna ou a sexta célula da primeira linha.
} 
Pascal dá uma demonstração para o terceiro princípio, mas, para nossos propósitos, não será necessário abordá-la aqui. Os primeiros dois princípios, em contraste, são intuitivamente óbvios.

Para ver como esses princípios se aplicam, investigaremos o mesmo exemplo dado acima, isto é, o caso em que faltam duas partidas para A e quatro para B. Ao fazer o cálculo será necessário fazer vários cálculos intermediários. Assim, suponha que falte apenas uma partida para A. Caso A ganhar a próxima partida, ele ganhará o jogo e, pelo primeiro princípio, ganhará a aposta toda, ou seja, $\frac{1}{1}$ da aposta. Caso A perder a próxima partida, faltará uma partida para cada jogador e, portanto, pelo segundo princípio, A ganhará $\frac{1}{2}$ da aposta. Agora, para aplicar o terceiro princípio, é necessário combinar as duas frações $\frac{1}{1}$ e $\frac{1}{2}$ da maneira indicada, sempre lembrando de que é necessário que as duas frações tenham o mesmo denominador. Desta forma, combinamos, da maneira indicada, as frações $\frac{2}{2}$ e $\frac{1}{2}$, resultando em $\frac{2+1}{2 \times 2}$, ou seja, $\frac{3}{4}$. Resumimos os cálculos da seguinte forma:

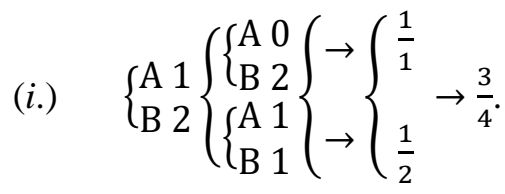

No que segue, daremos os cálculos intermediários apenas de forma resumida.

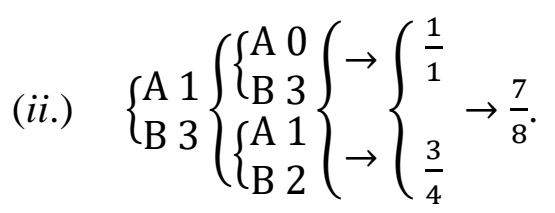

Observamos que, no penúltimo passo de (ii.), usamos o resultado do (i.). O uso de resultados anteriores acontecerá sempre nos seguintes cálculos. Faremos isso sem chamar atenção ao mesmo. 


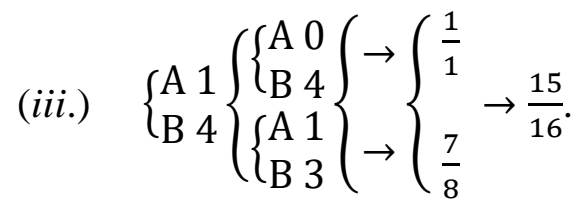

$$
\begin{aligned}
& \text { (vi.) }\left\{\begin{array} { l } 
{ \text { A } 2 } \\
{ \text { В } 3 }
\end{array} \{ \begin{array} { l } 
{ \text { A } 1 } \\
{ \text { B } 3 } \\
{ \text { A } 2 } \\
{ \text { B } 2 }
\end{array} \} \rightarrow \left\{\begin{array}{l}
\frac{7}{8} \\
\frac{1}{2}
\end{array} \rightarrow \frac{11}{16}\right.\right. \text {. }
\end{aligned}
$$

Finalmente, temos:

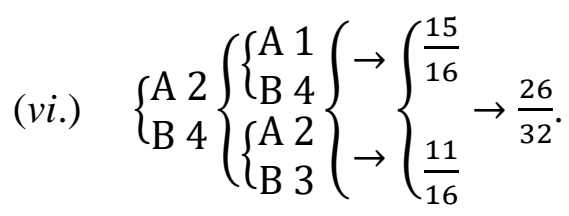

Desta forma, A receberá $\frac{26}{32}$, ou seja, $\frac{13}{16}$ dos 200 reais apostados. Consequentemente, B receberá $\frac{3}{16}$ da aposta. Observamos que isso é o mesmo resultado obtido usando o Triângulo Aritmético. Observamos ainda que o exemplo mostra o interesse de Pascal em querer usar o Triângulo Aritmético para determinar a divisão da aposta em jogos interrompidos. O procedimento usando os três princípios envolve longos cálculos intermediários ou, talvez, a elaboração de extensivas tabelas resumindo os resultados desses cálculos. Em contraste, o procedimento usando o Triângulo Aritmético é relativamente fácil e rápido.

Agora voltemos a nossa atenção para a demonstração de Pascal do seu procedimento de usar o Triângulo Aritmético para fazer a referida divisão.

\section{A Demonstração}

À primeira vista, a demonstração do procedimento é um tanto complicada, pois contém um número infinito de casos. Mas Pascal (2013, p. 92) aponta uma saída:

Embora essa proposição tenha uma infinidade de casos, demonstrá-la-ei, não obst

O $1^{\circ}$, a segunda base contém as partes de jogadores para quem faltam, ao todo, duas partidas. 
O $2^{\circ}$, se uma base qualquer contém as partes dos para quem falta a mesma quantidade de partidas quanto há de células, a próxima base terá a mesma propriedade, isto é, também conterá as partes dos jogadores para quem falta a mesma quantidade de partidas quanto há de células.

Disto se conclui, de imediato, que todas as bases do Triângulo aritmético têm a referida propriedade: pois, a segunda a tem pelo primeiro lema; assim, pelo segundo lema, a terceira a tem também e, em conseqüência, a quarta; e assim até a infinidade.

O que queríamos demonstrar.

A proposta de Pascal é uma formulação claríssima da Indução Matemática. De fato, Pascal foi creditado, por muito tempo, como sendo o originador desse tipo de demonstração. Hoje, sabemos que há precursores, mas parece que a sua formulação clara foi obra dele mesmo. Em contraste, muitos matemáticos da época usavam demonstrações por "indução" (mais propriamente chamada de "por enumeração"), que consistia em demonstrar algo por dois ou três casos e, em seguida, concluir que valia para todos os casos. Na verdade, Pierre de Fermat (1601-1665) criticou várias demonstrações de John Wallis (1616-1703) neste sentido. Ademais, é interessante relembrar que Fermat foi o originador da demonstração "por descida infinita" - um tipo de indução inversa. Nela, demonstra-se que se uma proposição vale para $n$, também vale para $n-1$, o que implica que o conjunto de números para os quais a proposição vale não tem mínimo, uma contradição óbvia nos casos em que a demonstração é bem-sucedida.

Voltando à demonstração de Pascal, vemos que só resta demonstrar os dois lemas.

O primeiro lema, ou seja, a base da indução, afirma que a proposição é verdadeira para a segunda diagonal. Isso corresponde ao caso em que falta a cada jogador uma partida para vencer o jogo. Reconhecemos intuitivamente que, nesse caso, cada jogador deverá ficar com metade da aposta. Aplicando a regra de Pascal, A receberá o que falta a $\mathrm{B}$ (1) sobre o total da diagonal (2), enquanto B receberá o que falta a A (1) sobre o mesmo total (2). Assim, o resultado, $\frac{1}{2}$ para cada jogador, conforme à nossa intuição.

É interessante observar que esse mesmo resultado intuitivo acontece sempre que falta o mesmo número de partidas para cada jogador. Com efeito, seja esse número $n$. Então, usa-se a $2 n$-ésima diagonal e, visto que a diagonal é simétrica em relação aos números contidos nas suas células, a soma dos primeiros $n$ desses números é igual à 
John A. Fossa

Aplicação do triângulo de Pascal

soma dos últimos $n$ deles. Assim, cada uma das referidas somas é igual a metade do total e cada jogador receberá metade da aposta.

Agora vejamos como Pascal (2013, p. 93-94) demonstra o passo da indução:

Se uma base qualquer, como a quarta, $\mathrm{D} \lambda$, contém as partes do jogador para quem faltam quatro partidas, isto é, se falta um para o primeiro e faltam três ao segundo, então a porção que pertencerá ao primeiro sobre a quantia que está em jogo é a quantia expressa pela seguinte fração: $\frac{\mathrm{D}+\mathrm{B}+\theta}{\mathrm{D}+\mathrm{B}+\theta+\lambda}$, cujo denominador é a soma das células da base e cujo numerador é as três primeiras; e se faltam duas partidas para um e duas para o outro, então a fração que pertencerá ao primeiro é $\frac{\mathrm{D}+\mathrm{B}}{\mathrm{D}+\mathrm{B}+\theta+\lambda}$; e se faltam três partidas para o primeiro e falta uma para o outro, a fração do primeiro é $\frac{\mathrm{D}}{\mathrm{D}+\mathrm{B}+\theta+\lambda}$, etc.

Então digo que a quinta base também contém as partes do jogador para quem faltam cinco partidas. E se faltam, por exemplo, duas partidas para o primeiro e três para o outro, a porção que pertence ao primeiro sobre a quantia que está em jogo é representada pela seguinte fração:

$$
\frac{\mathrm{H}+\mathrm{E}+\mathrm{C}}{\mathrm{H}+\mathrm{E}+\mathrm{C}+\mathrm{R}+\mu} .
$$

Pois, para saber o que pertence aos dois jogadores, para cada um dos quais faltam quaisquer partidas, basta, pelo lema anterior, tomar a fração que pertenceria ao primeiro, caso ganhasse, e a que lhe pertenceria, caso perdesse, convertê-las ao mesmo denominador, se estiveram diferentes, e formar a fração cujo numerador é a soma dos outros dois numeradores e cujo denominador é o dobro do precedente.

Vamos examinar, então, as frações que pertenceriam ao nosso primeiro jogador nos casos de ganho e perda.

Se o primeiro, para quem faltam duas partidas, ganhar a partida a ser jogada, só lhe faltará mais uma partida, faltando ainda três para o outro. Então, lhes faltarão quatro ao todo e, por hipótese, suas partes são achadas na quarta base, sendo que pertencerá ao primeiro a seguinte fração: $\frac{\mathrm{D}+\mathrm{B}+\theta}{\mathrm{D}+\mathrm{B}+\theta+\lambda}$.

Se, ao contrário, o primeiro perder, lhe faltarão ainda duas jogadas e somente duas para o outro. Assim, por hipótese, a fração do primeiro será $\frac{\mathrm{D}+\mathrm{B}}{\mathrm{D}+\mathrm{B}+\theta+\lambda}$. Logo, caso se separarem, pertencerá ao primeiro a seguinte fração:

$$
\frac{\mathrm{D}+\mathrm{B}+\theta+\mathrm{D}+\mathrm{B},}{2 \mathrm{D}+2 \mathrm{~B}+2 \theta+2 \lambda,} \frac{\text { ou seja, }}{\text { ou seja, }}, \frac{\mathrm{H}+\mathrm{E}+\mathrm{C}}{\mathrm{H}+\mathrm{E}+\mathrm{C}+\mathrm{R}+\mu}
$$

Pascal quer que contemplemos uma diagonal ("base", na terminologia dele) qualquer do Triângulo. Suponhamos, segundo Pascal, que a referida diagonal satisfaz o 
procedimento do Triângulo Aritmético para a divisão da aposta. Assim, será necessário demonstrar que a próxima diagonal também o satisfaz.

Aqui Pascal põe de lado qualquer atitude mais abstrata, tomando como exemplo, a quarta diagonal. Visto que a suposição então é que a quarta diagonal satisfaz o procedimento, ele dá as frações do que o primeiro jogador receberá caso falta(m) a ele 1, 2 e 3 partidas, dando essas frações pelas nomes (uma mistura de letras latinas e gregas) das células. Assim, sua tarefa se reduz a mostrar que a quinta diagonal também satisfaz o procedimento. Para tanto, deveria mostrar o que acontece nos casos em que falta $(m)$ ao primeiro jogador 1, 2, 3 e 4 partidas. Ele considera, no entanto, somente um caso: o em que faltam duas partidas ao primeiro jogador e, em consequência, três ao segundo jogador.

Para determinar a divisão no referido caso, Pascal usa o método explicado na seção anterior do presente trabalho, ou seja, determina o que o primeiro jogador, A, receberá caso ganhar e caso perder a próxima partida.

Seja primeiro que A ganhasse a próxima partida. Assim, faltará a ele uma partida, enquanto faltarão ao segundo jogador, B, três partidas. O total de partidas, portanto, serão quatro e podemos usar a quarta diagonal do Triângulo para determinar a parte que A deverá receber, pois, pela hipótese da indução, a quarta diagonal satisfaz o procedimento do Triângulo. Pelo referido procedimento, A receberá a fração representada por

$$
\frac{x}{z}=\frac{\mathrm{D}+\mathrm{B}+\theta}{\mathrm{D}+\mathrm{B}+\theta+\lambda}
$$

Seja, então, que A perdesse a próxima partida. Assim, faltarão a ele duas partidas, enquanto também faltarão duas partidas para B. De novo, o total das partidas são quatro e podemos usar a quarta diagonal para calcular a fração

$$
\frac{y}{z}=\frac{\mathrm{D}+\mathrm{B}}{\mathrm{D}+\mathrm{B}+\theta+\lambda}
$$

A parte da aposta que pertencerá a A, portanto, é 


$$
\frac{x+y}{2 z}=\frac{\mathrm{D}+\mathrm{B}+\theta+\mathrm{D}+\mathrm{B}}{2(\mathrm{D}+\mathrm{B}+\theta+\lambda)}
$$

Podemos expressar essa fração como

$$
\frac{\mathrm{D}+(D+B)+(B+\theta)}{2(\mathrm{D}+\mathrm{B}+\theta+\lambda)} .
$$

Então, visto que $\mathrm{D}=\mathrm{H}=1 \mathrm{e}$, pela lei de formação do Triângulo, $\mathrm{D}+\mathrm{B}=\mathrm{E}$ e $\mathrm{B}+\theta$ $=\mu$, temos

$$
\frac{\mathrm{H}+E+C}{2(\mathrm{D}+\mathrm{B}+\theta+\lambda)}
$$

Finalmente, observamos que a soma dos números das sucessivas diagonais são iguais às sucessivas potências de 2 . Para ver isso, consideremos a expansão do binômio

$$
(x+y)^{n}=a_{0} x^{n}+a_{1} x^{n-1} y+a_{2} x^{n-2} y^{2}+\cdots+a_{n} y^{n} .
$$

Em particular, pondo $x=y=1$, obtemos

$$
2^{n}=(1+1)^{n}=a_{0}+a_{1}+a_{2}+\cdots+a_{n},
$$

onde o último membro da equação é, claramente, a soma dos coeficientes da expansão do binômio, o que é dada pelo Triângulo Aritmético. Em consequência, a fração que representa a parte da aposta que pertence a A, no caso em que faltam duas partidas para A e três para $\mathrm{B}$, se reduz a

$$
\frac{H+E+\mathrm{C}}{H+E+C+R+\mu} .
$$

Mas essa fração é a fração dada pelo procedimento do Triângulo Aritmético para determinar a divisão da aposta no referido caso. 


\title{
A Validade da Demonstração de Pascal
}

Em conclusão, devemos fazer uma apreciação da validade da demonstração de Pascal. Em primeiro lugar, mencionamos, de novo, que a formulação do argumento por indução matemática feita por Pascal é impecável. A sua demonstração da base da indução também está sem problemas. A demonstração do passo da indução, no entanto, é um tanto problemática.

Observamos que o fato de que Pascal usa os nomes das células, em vez dos números nelas contidos, não garante a generalidade necessária para uma demonstração bem-sucedida. De fato, a demonstração proposta somente mostra que a proposição valerá para a quinta diagonal, se valer para a quarta - e mesmo aqui a demonstração é feita somente para um elemento da diagonal, em vez de todos os elementos dessa diagonal. Mas, o necessário é fazer a demonstração geral de que a proposição valerá para a $n+1$-ésima diagonal, se valer para a $n$-ésima diagonal. Pelos padrões modernos, portanto, o argumento não é válido.

Logo depois da demonstração, porém, Pascal (2013, p. 94, itálico no original) adiciona a seguinte explanação sobre a generalidade do seu argumento:

\begin{abstract}
Isto é demonstrado da mesma forma sobre todas as outras bases, sem qualquer diferença, visto que o fundamento da demonstração é que uma base é sempre o dobro da precedente pela sétima conseqüência e que, pela décima conseqüência, tantas células ${ }^{3}$ que quiser de uma base são iguais às mesmas tantas da base precedente (que é sempre o denominador da fração no caso de ganho), mas as mesmas células, menos uma, (que é o numerador da fração no caso de perda). Visto que isto é verdadeiro geralmente no triângulo todo, a demonstração será sempre sem problema e universal.
\end{abstract}

Desta forma, fica evidente que Pascal, na sua demonstração, estava tentando dar um template que se aplicaria ao Triângulo todo, modelando o argumento que poderia ser usado para qualquer caso específico. O problema com esse tipo de argumento é que não demonstra o que o modelo afirma, mas apenas apela ao leitor a reconhecer, intuitivamente, a sua aplicação generalizada.

Parte do problema é a pobreza do simbolismo usado por Pascal. Sugerimos o uso do simbolismo $c_{i j}$ para representar a célula (ou o número nela contido) da $i$-ésima diagonal e a j-ésima coluna. Os elementos da $n$-ésima diagonal então serão dados por

\footnotetext{
${ }^{3}$ Isto é, a soma dos números dessas células.
} 
John A. Fossa

Aplicação do triângulo de Pascal

$c_{n j}$, com $n$ fixo e $1 \leq j \leq n$. Deixaremos como exercício para o leitor tentar elaborar, seguindo a ideia fundamental de Pascal, uma demonstração generalizada do procedimento do Triângulo Aritmético para a divisão da aposta. ${ }^{4}$

\section{Referência}

PASCAL, Blaise. Tratado sobre o Triângulo Aritmético. Trad. de John A. Fossa e Fabricio Possebon. Natal: EDUFRN, 2013.

\footnotetext{
${ }^{4}$ Agradeço à colega Enne Karol Venâncio de Sousa pelas sugestões referentes à correção gramatical do presente trabalho.
} 